\title{
A fully three-dimensional model of the interaction of driven elastic filaments in a Stokes flow with applications to sperm motility
}

\author{
Julie Simons $^{\mathrm{a}}$, Lisa Fauci ${ }^{\mathrm{a}}$, Ricardo Cortez ${ }^{\mathrm{a}}$ \\ ${ }^{a}$ Center for Computational Science and Mathematics Department, Tulane University, \\ 6823 St. Charles Ave., New Orleans, LA 70118, USA
}

\section{Abstract}

In many animals, sperm flagella exhibit primarily planar waveforms. An isolated sperm with a planar flagellar beat in a three-dimensional unbounded fluid domain would remain in a plane. However, because sperm must navigate through complex, three-dimensional confined spaces along with other sperm, forces that bend or move the flagellum out of its current beat plane develop. Here we present an extension of previous models of an elastic sperm flagellar filament whose shape change is driven by the pursuit of a preferred curvature wave. In particular, we extend the energy of the generalized elastica to include a term that penalizes out-of-plane motion. We are now able to study the interaction of free-swimmers in a 3D Stokes flow that do not start out beating in the same plane. We demonstrate the three-dimensional nature of swimming behavior as neighboring sperm swim close to each other and affect each others' trajectories via fluid-structure coupling.

Keywords: Sperm motility, three-dimensional flagellum, planar waveforms,

Email addresses: jsimons@tulane.edu (Julie Simons), fauci@tulane.edu (Lisa Fauci), rcortez@tulane.edu (Ricardo Cortez) 
Stokes equations, regularized Stokeslets

\section{Introduction}

For successful fertilization, sperm must be able to navigate complex environments to reach the egg. Thus, sperm motility patterns are vital components of sexual reproduction. In many species, sperm flagellar waveforms are planar or near-planar, though helical waveforms are also stable [48, 51]. Planar waveforms of an isolated beating flagellum result in trajectories that are characterized by either linear or circular paths within the plane, depending on the symmetry of the beat pattern [36, 52]. However, forces exerted on the planar swimmer by proximal surfaces or nearby swimmers can easily bend or pull part or all of the flagellum out of its current beat plane.

Three-dimensional behavior of sperm flagella has been experimentally observed [50] and some species exhibit "figure 8" or helical deviations of the waveform out of the plane $[10,51]$. More recent experimental advances in tracking sperm at a high resolution have exposed the three-dimensional nature of swimming trajectories of sperm in populations, providing experimental evidence for helical or "chiral ribbon" trajectories $[7,42,43]$. The recent review of Guerrero et al. [21] highlights the importance of understanding sperm motility in fully three-dimensional settings in order to correctly characterize and assess fertility in sperm.

The hydrodynamics of sperm motility is characterized by low Reynolds number, viscous fluid mechanics, and has been the inspiration of many classic studies in biological fluid dynamics [15], including G.I. Taylor's swimming sheet [46] and the resistive force theory of Gray and Hancock [20]. In these 
early models, the kinematics of the flagellar waveform were imposed, and the resulting swimming speed of the flagellum was calculated. Recognizing that a sperm flagellum is an elastic structure whose realized kinematics are determined by its coupling to the surrounding viscous fluid, models have been developed that treat the flagellum as a generalized Euler elastica, whose shape change is driven by the pursuit of a preferred curvature wave [17]. Such flagellar models, essentially of swimming sheets, have been coupled to a two-dimensional fluid and have been used to study synchronization of finite swimmers and their attraction to walls [16], and the effect of viscoelasticity on flagellar swimming $[6,47]$. More recently, these driven-elasticas have been coupled to a model for three-dimensional Stokes flow and have been used to study the emergence of a hyperactivated waveform due to calcium dynamics in a single, isolated flagellar filament [34], and the elastic attachment of a planar flagellar filament to an orthogonal planar wall [40]. While the dynamics of these filaments were coupled to a three-dimensional domain of fluid, the symmetry imposed by a single flagellum confined to a plane in free-space or one beating perpendicular to an infinite planar wall does not result in any out-of-plane component of force on the flagellum.

Motivated by the understanding sperm-sperm and sperm-boundary interactions in more general settings, even for driven filaments that pursue a planar waveform, here we extend the generalized Euler elastica energy formulation of [17] to include a penalty for out-of-plane motion. The native flagellar plane will evolve in time. The energy that drives this new model is rotationand translation-invariant, therefore the filament will be a free-swimmer that generates zero total force and torque. 
While several different mathematical approaches may be used to couple flagellar forces to a fluid, here we use the 3D method of regularized Stokeslets [9]. In the following sections, we present the mathematical formulation of the three-dimensional model, how to incorporate multiple nearby sperm, and then discuss the implications for three-dimensional trajectories and behaviors in sperm populations.

\section{Methods}

\subsection{Derivation of the Three-Dimensional Model}

We model the sperm flagellum as a three-dimensional curve $\mathbf{X}(s, t)$ that exerts forces along its length in a viscous fluid governed by the incompressible Stokes equations:

$$
\begin{aligned}
& \mu \Delta \mathbf{u}=\nabla p-\int_{0}^{L} \mathbf{f}(\mathbf{X}(s, t), t) \phi_{\epsilon}(\mathbf{x}-\mathbf{X}(s, t)) d s \\
& \nabla \cdot \mathbf{u}=0
\end{aligned}
$$

where $L$ is the total length of the flagellum, $s$ is arc length, $t$ is time, $\mu$ is the dynamic viscosity, $\mathbf{u}$ is fluid velocity, and $p$ is the pressure. The forces $\mathbf{f}(\mathbf{X}, t)$ exerted by the flagellum upon the surrounding fluid are regularized by a blob function $\phi_{\epsilon}$ defined by:

$$
\phi_{\epsilon}(r)=\frac{15 \epsilon^{4}}{8 \pi\left(r^{2}+\epsilon^{2}\right)^{7 / 2}} .
$$

This is a regularized delta function that distributes the force in a small fluid volume around the curve $[8,9]$. This regularization eliminates singularities in the velocity field and the parameter $\epsilon$ is chosen to be on the order of the diameter of the sperm flagellum. The flagellum will be considered an 
immersed boundary that moves with the fluid velocity at all points along its length.

Our model assumes that sperm flagella have a nearly planar beat. Both helical and planar flagellar beat forms may be stable. Planar waveforms may be more prevalent near surfaces and observed helical waveforms may in fact be more aptly described as "flattened helices" [4, 37, 51]. The planar or quasi-planar nature of the sperm flagellum is most likely controlled by the structure of the microtubule doublets and dynein bridges of the axoneme [19, $22,26,27,35,39]$. We do not seek to model the underlying structure of the flagellum nor how a planar preference might arise, but instead conceptualize the sperm flagellum as a thin filament that would naturally have a planar shape in the absence of external forces.

This extended model is formulated so that a flagellum can accommodate three-dimensional effects that cause deviations from planar configurations. These effects could include surfaces, other sperm swimming nearby, or other external forces. To accomplish this, we incorporate forces that restrict outof-plane deviations of the flagellum. Within the flagellar plane, we prescribe curvature-based forces along the flagellum as in [17], which enable the sperm to move in a wave-like manner.

The forces $\mathbf{f}$ will be derived using a continuous energy formulation that is an extension of the internally-actuated filament model in [17]. In order to define the energy, we first define a local coordinate system in which the flagellar plane is $z=0$. This will be referred to as the flagellum frame of reference. In this frame of reference, bending forces that modulate the swimming behavior of the flagellum will occur in the plane $z=0$ alone. 
Moreover, non-planar deviations can be understood as deviations in the $z$ component and forces restricting these deviations can be found. For clarity, in the flagellum frame of reference, we refer to the Cartesian coordinates as $\hat{x}, \hat{y}$, and $\hat{z}$ and the coordinates of the flagellum itself as $\hat{\mathbf{X}}$. Thus the flagellar plane is the plane $\hat{z}=0$.

Using this notation, we define the total energy as:

$$
\mathcal{E}(\hat{\mathbf{X}}, t)=\mathcal{E}_{\text {tens }}(\hat{\mathbf{X}}, t)+\mathcal{E}_{\text {bend }}(\hat{\mathbf{X}}, t)+\mathcal{E}_{\text {plane }}(\hat{\mathbf{X}}, t)
$$

where $\mathcal{E}_{\text {tens }}$ is a tensile energy term that enforces inextensibility, $\mathcal{E}_{\text {bend }}$ is a time-dependent bending energy that drives the beating behavior of the filament, and $\mathcal{E}_{\text {plane }}$ is the energy arising from out-of-plane deviations. Each energy is defined as follows:

$$
\begin{aligned}
& \mathcal{E}_{\text {tens }}(\hat{\mathbf{X}}, t)=\frac{1}{2} S_{t} \int_{0}^{L}\left[\left\|\frac{\partial \hat{\mathbf{X}}}{\partial s}\right\|-1\right]^{2} d s \\
& \mathcal{E}_{\text {bend }}(\hat{\mathbf{X}}, t)=\frac{1}{2} S_{b} \int_{0}^{\ell}\left[\left(\frac{\partial \hat{\mathbf{X}}}{\partial \alpha} \times \frac{\partial^{2} \hat{\mathbf{X}}}{\partial \alpha^{2}}\right) \cdot \mathbf{e}_{3}-C(\alpha, t)\right]^{2} d \alpha \\
& \mathcal{E}_{\text {plane }}(\hat{\mathbf{X}}, t)=\frac{1}{2} S_{p} \int_{0}^{L}\left[\frac{\partial \hat{\mathbf{X}}}{\partial s} \cdot \mathbf{e}_{3}\right]^{2} d s
\end{aligned}
$$

where $S_{i}$ are stiffness coefficients, $L$ is the length of the flagellum, and $\mathbf{e}_{3}=$ $(0,0,1)^{T}$ is the normal to the flagellar plane. The function $C(\alpha, t)$ is the preferred curvature that drives the swimming motion of the sperm. Since this function is restricted to the plane $\hat{z}=0$, the parameter $\alpha$ refers to the projection of the filament arc length onto that plane, and $\ell$ is the total projected arc length in that plane. We take $\ell$ to be approximately constant because deviations from the plane are assumed to be small. 
With these definitions, the energies (2) and (3) are the same energies described in [17] and the energy (4) is the new addition to our model. The energy (4) is non-zero when there are local deviations of the flagellum in the $\hat{z}$ direction (i.e. deviations from the flagellar plane). The total energy $\mathcal{E}$ is translation- and rotation-invariant and forces in the flagellum frame of reference $\hat{\mathbf{f}}$ can be calculated as

$$
\hat{\mathbf{f}}=-\frac{\partial \mathcal{E}}{\partial \hat{\mathbf{X}}}
$$

These resulting forces seek to minimize the total energy, where the tensile, bending and planarity stiffness constants $\left(S_{t}, S_{b}, S_{p}\right)$ determine how closely the preferred planar traveling wave is realized. In particular, the tensile stiffness $S_{t}$ is chosen large enough so that the filament is effectively inextensible, the bending stiffness $S_{b}$ reflects the bend modulus of the flexible filament, and the planarity stiffness $S_{p}$ controls the penalty for deviation from the plane. Finding the forces $\mathbf{f}$ in the original frame of reference requires a transformation from the $(\hat{x}, \hat{y}, \hat{z})$ coordinates to the global Cartesian coordinates $(x, y, z)$.

For computational purposes, we will discretize the integral in (1) by defining discrete points $\mathbf{X}_{k}$ along the flagellum (where $k$ denotes the index of the point). Figure 1 shows a schematic of the physical domain of the discretized flagellum for a preferred sinusoidal waveform with amplitude $b$.

Using the method of regularized Stokeslets described in [9], the fluid velocity is given by

$$
\mathbf{u}(\mathbf{x})=\sum_{k} \frac{r_{k}^{2}+2 \epsilon^{2}}{\left(r_{k}^{2}+\epsilon^{2}\right)^{3 / 2}} \mathbf{f}_{k}+\frac{\left(\mathbf{f}_{k} \cdot\left(\mathbf{x}-\mathbf{X}_{k}\right)\right)\left(\mathbf{x}-\mathbf{X}_{k}\right)}{\left(r_{k}^{2}+\epsilon^{2}\right)^{3 / 2}}
$$


where $r_{k}=\left\|\mathbf{x}-\mathbf{X}_{k}\right\|$ and $\epsilon$ is set to be close to the flagellum diameter. The points will be discretized so that their spacing is less than $\epsilon$. The discrete forces $\mathbf{f}_{k}$ define the forces the flagellum exerts at points $\mathbf{X}_{k}$, and are calculated from discretized versions of the energies (2)-(4). The motion of the flagellum points is described by the equation

$$
\left.\frac{d \mathbf{X}_{k}}{d t}=\mathbf{u}\left(\mathbf{X}_{k}(t), t\right)\right)
$$

which is approximated using a forward Euler method. In the next several sections, we describe the specific components of the three-dimensional preferred curvature model.

\subsection{Derivation of the discrete flagellar plane bending energy}

We discretize the bending energy (3), as in [17]:

$E_{b e n d, x y}=\frac{1}{2} S_{b} \sum_{k=2}^{N-1}\left(\frac{\left(\hat{x}_{k+1}-\hat{x}_{k}\right)\left(\hat{y}_{k}-\hat{y}_{k-1}\right)-\left(\hat{y}_{k+1}-\hat{y}_{k}\right)\left(\hat{x}_{k}-\hat{x}_{k-1}\right)}{\Delta \alpha^{3}}-C_{k}(t)\right)^{2} \Delta \alpha$

where $S_{b}$ is the bending stiffness constant, $N$ is the total number of points along the flagellum, and $\Delta \alpha$ is a constant equal to the desired spacing between the points $\left(\hat{x}_{k}, \hat{y}_{k}, 0\right)$. The preferred curvature $C_{k}(t)$ is a timedependent sinusoidal function similar to the one used in [17]:

$$
C_{k}(t)=\kappa^{2} b \sin \left(\kappa \alpha_{k}-\omega t\right)
$$

where $\alpha_{k}$ is the arc length distance along the flagellum (projected onto the plane $\hat{z}=0)$ from the head point $\left(\hat{x}_{1}, \hat{y}_{1}, 0\right)$ up to the point $\left(\hat{x}_{k}, \hat{y}_{k}, 0\right)$, and $\kappa$ and $\omega$ are the wavenumber and frequency parameters, respectively. In this model, we choose $\kappa=\frac{2 \pi}{L}$ so there is approximately one wavelength per flagellum length, but our results are not qualitatively sensitive to this choice. 
Small out-of-plane deviations of the flagellum imply that $\Delta \alpha$ may be smaller but not too different from $\Delta s$ (the desired spacing between points in 3D). In order not to overestimate the desired planar curvature forces for three-dimensional flagellar configurations, we consider the three-dimensional vector of length $\Delta s$ given by

$$
\mathbf{r}_{k+1}=\Delta s \frac{\hat{\mathbf{X}}_{k+1}-\hat{\mathbf{X}}_{k}}{\left\|\hat{\mathbf{X}}_{k+1}-\hat{\mathbf{X}}_{k}\right\|},
$$

and define $\Delta \alpha_{k+1}$ as the length of the projection of vector $\mathbf{r}_{k+1}$ onto the flagellar plane:

$$
\Delta \alpha_{k+1}:=\left\|\mathbf{r}_{k+1}-\left(\mathbf{e}_{3} \cdot \mathbf{r}_{k+1}\right) \mathbf{e}_{3}\right\|
$$

With this definition, the factors of $\Delta \alpha^{3}$ and $\Delta \alpha$ in Eq. (5) are replaced with

$$
\Delta \alpha^{3} \rightarrow \Delta \alpha_{k} \Delta \alpha_{k+1} \frac{\Delta \alpha_{k}+\Delta \alpha_{k+1}}{2}, \quad \Delta \alpha \rightarrow \frac{\Delta \alpha_{k}+\Delta \alpha_{k+1}}{2}
$$

We note that $\Delta \alpha_{k}=\Delta s$ for all $k$ when the points are exactly in the plane $\hat{z}=0$.

\subsection{Plane restriction force}

To address out-of-plane deviations, we discretize the energy (4) as:

$$
E_{\text {plane }}=\frac{1}{2} S_{p} \sum_{k=1}^{N-1}\left(\frac{\hat{z}_{k+1}-\hat{z}_{k}}{\Delta s}\right)^{2} \Delta s .
$$

This energy formulation restricts any non-planar deviations via forces in the $\hat{z}$-direction but is a local effect that seeks to minimize the total quadratic variation in $\hat{z}$, hence reducing the concavity of $\hat{z}(s)$. The force derived from this energy acting upon an interior point $k \in(2, \ldots, N-1)$ would then be:

$$
\hat{\mathbf{f}}_{k}=S_{p}\left(\hat{z}_{k+1}-2 \hat{z}_{k}+\hat{z}_{k-1}\right) \frac{1}{\Delta s} \mathbf{e}_{3} \approx S_{p} \Delta s \hat{z}^{\prime \prime}\left(s_{k}\right) \mathbf{e}_{3} .
$$


Because the energy function $E_{\text {plane }}$, like the bending and tensile energies, is rotationally- and translationally-invariant, the net force and the net torque due to $E_{\text {plane }}$ satisfy:

$$
\sum_{k} \hat{\mathbf{f}}_{k}=\sum_{k} \hat{\mathbf{X}}_{k} \times \hat{\mathbf{f}}_{k}=\mathbf{0} .
$$

This can be proven by direct calculation from (8) and is due to Noether's Theorem [1]. These two conditions on the net force and net torque are constraints required for self-propelled organisms.

\subsection{Incorporating asymmetry}

Motility patterns in sperm are typically characterized as active (symmetric) or hyperactive (asymmetric with high amplitude). Because hyperactivity is required for successful fertilization of the egg $[44,45,52]$, we consider both asymmetric and symmetric preferred beat-forms. To incorporate asymmetry into this model, we use a simple amplitude modulation approach as in [34] to set the preferred curvature function $C_{k}(t)$ in expression (6) along the flagellum for all times $t$. For our flagellum of length $100 \mu \mathrm{m}$, we let the amplitude $b$ of the curvature function (6) take the following values:

$$
b= \begin{cases}b_{1} & \text { if } \sin \left(\kappa s_{k}-\omega t\right)<0 \\ b_{2} & \text { if } \sin \left(\kappa s_{k}-\omega t\right) \geq 0\end{cases}
$$

In the case of a symmetric waveform, we choose $b_{1}=b_{2}=10 \mu \mathrm{m}$. To explore the effect of a weak asymmetry in curvature, we choose $b_{1}=10 \mu \mathrm{m}$ and $b_{2}=$ $15 \mu \mathrm{m}$. For a stronger, higher amplitude asymmetry we choose $b_{1}=10 \mu \mathrm{m}$ and $b_{2}=25 \mu \mathrm{m}$. The stronger asymmetry parameters have been chosen such that the behavior is closer to the deeper bends characteristic of hyperactive sperm motility patterns (see previous models in $[34,40]$ ). 


\subsection{Defining the Flagellum Frame of Reference}

At each time step, we need to update the preferred flagellar plane and flagellar frame of reference. We use the method of least squares, as described in [12], to define this plane. Given discrete points along the flagellum $\mathbf{X}_{k}$, we find the center of mass of the flagellum, denoted by $\overline{\mathbf{X}}$. This is simply the average position of all points $\mathbf{X}_{k}$. We define the flagellar plane as the plane that minimizes the sum of the squares of the orthogonal distances between the centered points $\mathbf{X}_{k}-\overline{\mathbf{X}}:=\left(x_{k}, y_{k}, z_{k}\right)^{T}$ and the plane. The plane is represented by the vector $\mathbf{v}=(a, b, c)^{T}$, corresponding to least squares fit plane $a x+b y+c z=0$. We note that because the points $\left(x_{k}, y_{k}, z_{k}\right)$ have zero average, this plane must go through the origin by construction.

The vector $\mathbf{v}$ defines the normal to the flagellar plane. This is equivalent to the $\hat{z}$ coordinate direction in the local (Lagrangian) flagellum frame of reference. Noting that a plane splits the space into two half-spaces, care is taken to ensure that $\mathbf{v}$ is always pointing towards the same half-space. This is important because the preferred curvature model in [17] requires the ability to discern the sign of the curvature. In other words, we must be able to choose between a plane normal vector pointing in the positive or negative $\hat{z}$ direction. Since our model is designed to represent a continuous process and we use a small numerical time step, the flagellar plane should not change significantly between time steps. Therefore, we add a check point in our algorithm that verifies that the normal vector from the previous time step and the normal vector from the current time step have a positive dot product.

Because forces within the flagellar plane are invariant under rotation and 
translation, we may choose any $\hat{x}$ and $\hat{y}$ directions that would result in an orthonormal triad with $\mathbf{v}$ with the appropriate "right-hand rule" relationship between the coordinate directions. Figure 2 shows an example of a threedimensional flagellum configuration, the least squares plane fit to the centered data, as well as the flagellum frame of reference.

\subsection{Computational Method}

In all simulations, each flagellum is initialized with a non-collinear configuration at time step $j=0$ to ensure that a unique flagellar plane can be defined. The method for computing flagellar positions, forces, and velocities over time is as follows:

1. Calculate center of mass of flagellum points $\mathbf{X}_{k}$ and translate data so they have a center of mass at the origin.

2. Using the method of least squares, find the flagellar plane for centered flagellar data. The normal to this plane is represented by the normal vector $\mathbf{v}_{j}$.

3. For time step $j>0$ : if $\mathbf{v}_{j} \cdot \mathbf{v}_{j-1}<0$, let $\mathbf{v}_{j}=-\mathbf{v}_{j}$ (signed curvature checkpoint).

4. Define $\hat{\mathbf{X}}_{k}$ using the flagellum frame of reference determined by $\mathbf{v}_{j}$.

5. Find forces $\hat{\mathbf{f}}_{k}$ for the points $\hat{\mathbf{X}}_{k}$ based on the total energy $\mathcal{E}$.

6. Rotate forces $\hat{\mathbf{f}}_{k}$ back to original frame of reference to obtain $\mathbf{f}_{k}$.

7. Solve for velocities $\mathbf{u}_{k}$ using the method of regularized Stokeslets and forces $\mathbf{f}_{k}$, as described in [34].

8. Move all points $\mathbf{X}_{k}$ using a forward Euler time step with velocities $\mathbf{u}_{k}$.

9. Let $j=j+1$ and repeat. 


\subsection{Multiple Sperm}

We are interested in how sperm trajectories are influenced by nearby neighbors. The model proposed here can consider any three-dimensional flagellum configuration with multiple sperm present. However, for realistic scenarios with multiple sperm, the model must not allow flagellum points to overlap. To address this, we add a repulsive force that acts on points that come within a certain distance of each other.

The repulsive force acting on the point $\mathbf{X}_{k}$ by the point $\mathbf{X}_{j}$ on separate flagellum will take the following form:

$$
\mathbf{g}_{k}^{j}=S_{r}\left(\frac{1}{d}-\frac{1}{\min (r, d)}\right)\left(\mathbf{X}_{k}-\mathbf{X}_{j}\right)
$$

where $r=\left\|\mathbf{X}_{k}-\mathbf{X}_{j}\right\|$ and $d$ is a fixed distance. We choose this form because it is continuous in $r$ and it tends to infinity as $r$ approaches zero, which ensures that points do not get too close to one another if the time step is small. The choice of parameter $S_{r}$ is arbitrary, but should be chosen so that the repulsion forces do not get too large too quickly (compared to the curvature forces) as $r$ falls below $d$. The forces $\mathbf{g}_{k}^{j}$ will be added to the forces $\mathbf{f}_{k}$ before solving for velocities in the computational method. Note that contributions from this repulsion force maintain force- and torque-free swimming.

\section{Results}

Here we explore the effects of extending the elastica model of driven filaments to include an energy contribution for out-of-plane motion. In particular, consider the cases of both a single planar flagellum swimming in 3D free-space, and a pair of coplanar flagella pursuing waves in that plane, whose 
initial configurations are all planar. In these cases, the energy contribution for out-of-plane motion is always zero, and the trajectories of the swimmers remain in the initial plane. However, a pair of flagella each pursuing planar waveforms in parallel planes will interact with each other and generate out-of-plane motion. Below we consider these cases, and also explore the consequences of out-of-plane perturbations of initial flagellar configurations in both an isolated flagellum and pairs of interacting flagella. All simulations use the model parameter values listed in Table 1 and were initialized with the flagellar configurations shown in Figure 3. The top row (Figure $3 \mathrm{a}-3 \mathrm{c}$ ) is used for coplanar or perturbed coplanar initial conditions, and the bottom row (Figure 3d-3f) is used for parallel plane initial configurations. From these small amplitude initial configurations (chosen for simplicity), the sperm flagellum evolves into its natural waveform over time. In all two sperm simulations presented here, the preferred curvature waves are specified to be in-phase. For further description of the method of regularized Stokeslets and scalings used for the fluid equations, please see $[8,40]$.

\subsection{Single Sperm}

We first consider a single flagellum that is pursuing a symmetric, planar wave, but whose initial small amplitude configuration is perturbed slightly out-of-plane. The flagellum was started with a primarily $x-y$ sinusoidal planar conformation, with the head of the sperm slightly raised from the $x-y$ plane (shown in black in Figure 3). We examine the dynamics of relaxation of this sperm flagellum to its native planar form from its initial three-dimensional configuration for various planarity stiffnesses $S_{p}$. The higher the value of this stiffness parameter, the faster one would expect the flagellum to relax 
towards its native plane. The relaxation to the native flagellar plane can be characterized by considering the maximum value of $\left|\hat{z}_{k}\right|$ over all flagellum points. Figure 4 shows the relaxation behavior of this quantity over time

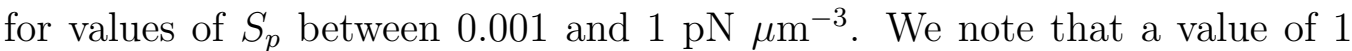
is of the same order of magnitude as the inextensibility parameter connecting the flagellar points together. With this parameter value, the flagellum approaches its native plane within $10^{-4} \mathrm{~s}$ (or $10^{-3}$ beats). For symmetric waveforms, a single sperm would relax to a planar configuration and swim linearly forward within that plane. For an asymmetric waveform, the sperm would also relax to a planar configuration and swim in a circular motion within its native plane, as shown in Figure 9a and Movie M1.

\subsection{Two Sperm}

We examine the cases of two identical flagella pursuing in-phase planar waves in (a) the same plane and (b) parallel planes. The initial configurations are taken to be perfectly planar, with no perturbations (Figures 3a-3c with no perturbation from $z=0$, and Figures $3 \mathrm{~d}-3 \mathrm{f})$. In the coplanar case, the sperm flagella attract and swim side by side, never leaving the plane $z=0$. The trajectories for this scenario are shown in Figure 5a and Movie M2. Woolley et al. [49] demonstrated this attraction between bull spermatozoa in experiments where the swimming was confined to a glass surface. Numerical simulations using multi-particle collision dynamics in two dimensions by Yang et al. [53] and bead models in three dimensions by Llopis et al. [28] also demonstrate the attraction of nearby flagella.

In contrast, when two flagella are initialized in parallel planes, the flagella repel over time. This is shown by the trajectories in Figure $5 \mathrm{~b}$ and the Movie 
M3, where the two sperm are initially placed half a flagellum length (50 $\mu \mathrm{m})$ apart. Below, we will see that when they are placed closer together initially, the flagella may attract before ultimately repelling and swimming away from each other.

The velocities achieved by two identical interacting symmetric swimmers, when compared to an identically-actuated single swimmer, depend on relative distance and orientations of the flagella. The velocities versus minimum distance between the two flagella as a function of time are plotted in Figure 6 for four different initial configurations of two swimmers that are pursuing identical symmetric waveforms, and whose planarity stiffness constant is $S_{p}=$ 1. Whereas a single swimmer has an average path velocity (i.e. the speed of the sperm over one beat period) of approximately $62 \mu \mathrm{m} / \mathrm{s}$, the coplanar swimmers (Figure 5a) that are attracted towards each other have a decreased velocity of $50 \mu \mathrm{m} / \mathrm{s}$ after they reach a steady swimming state (see Figure 6c). The swimmers that start $50 \mu \mathrm{m}$ apart in parallel planes (see Figure 5b) swim slower at all times, and approach a single swimmer's speed as they swim away from each other, as shown in Figure 6a. If the distance between initially parallel swimmers is reduced from $50 \mu \mathrm{m}$ to $5 \mu \mathrm{m}$, the swimmers actually see a transient increase in velocity and attract towards each other. They eventually do swim away from each other, however, even without a repelling force, as demonstrated in Figure 6b. The initial attraction of parallel swimmers, rotating their native plane and leading to an effective repulsion at longer times, is consistent with the observations of Llopis et al. [28].

Now we are going to consider perturbed coplanar initial configurations, 
as depicted in Figures 3a-3c. Figure 7 through 9 and Movies M4-M7 show examples of two sperm swimming near each other with variations in the symmetry of the preferred curvature waveform. In particular, Figure 7 shows the relative motions of the sperm bodies for the two symmetric swimmers over a period of 10 seconds. Initially, the two sperm are swimming side-by-side in slightly different planes (labeled by $t=0.1$ seconds). As time progresses, the sperm are attracted towards each other (labeled by $t=5.1$ seconds) and then move away from each other (labeled by $t=10.1$ seconds). The trajectories of the heads of these two swimmers are shown in Figure Figure 8a. While near each other, the fluid-structure coupling of the two sperm causes each filament to impact the trajectory (and preferred flagellar plane) of the other. The supplementary Movie M4 shows the swimming behavior of this pair of perturbed coplanar swimmers.

Although two perfectly coplanar swimmers pursuing a planar waveform would remain in that plane, this simulation shows that a small initial perturbation from that plane is never recovered. As is noted in Llopis et al. [28], coplanar swimming is an unstable configuration. Figure 8 shows that even with symmetric motility patterns, fully three-dimensional trajectories may appear. We also see that these trajectories depend upon the intrinsic properties of the the actuated swimmer, since different planarity stiffness constants result in different trajectories. For the stiffness parameter values shown, it appears that a weaker planar restriction stiffness results in longerterm cooperativity, as the flagella remain close to each other for a longer period of time. This period of close swimming shows that symmetricallybeating sperm in different planes may move in wave-like patterns, weaving 
around each other before eventually swimming away. Velocities for these swimmers decrease as they get closer to each other, but eventually increase towards that of a single swimmer as they swim apart (see Figure 6d). It appears that for transient periods of time, these swimmers may swim faster than a single swimmer due to their evolving relative orientation.

For a single flagellum, an asymmetric waveform acting upon a planar flagellum would result in a circular trajectory within the flagellar plane alone. Figure 9 shows that for two sperm, weak asymmetry in the waveform can lead to more complex three-dimensional trajectories. The flagellar plane of each individual sperm is rotated due to the presence of a nearby neighbor. Thus, spiral or pseudo-helical ("slinky"-like) trajectories can evolve from asymmetric waveforms. Movie M6 shows this swimming behavior over time. Movie M7 show high amplitude, strongly asymmetric waveforms that would characterize hyperactivity. Such high asymmetry and amplitude cause neighboring sperm to swim in more ball-like trajectories.

\section{Conclusions}

Mathematical and computational models have addressed various aspects of the hydrodynamic interaction of flagella, from infinite sheets $[6,13,14$, 46] to more recent investigations in three dimensions [28, 29], along with novel laboratory experiments [5]. Here we have presented an extension of the driven elastica model of a swimming flagellum [17] into a fully threedimensional formulation that penalizes out-of-plane waveforms. This allows the investigation of the fluid dynamic interaction of nearby swimmers, whose preferred planar waveforms may be altered by the flow. 
This model is able to exhibit fully three-dimensional, helical and ball-like trajectories arising from simple planar waveforms due to fluid-structure coupling of nearby swimmers. In mammalian sperm, hyperactivity is thought to facilitate reorientation of the sperm body so that sperm can chemotactically move towards the egg [25]. The ball-like trajectories observed in our simulations of hyperactive waveforms may be functionally equivalent to the tumbling state often described in bacterial chemotaxis, which enables bacteria to move towards favorable environments by randomly reorienting themselves before swimming in a new direction [2]. These trajectories, indeed, may be a benefit of complex environments and neighboring swimmers, in that they enable individual sperm to reorient in a fully three-dimensional space instead of just within their natural plane.

Cooperativity has been established in rodent sperm, where sperm cells swim together in the form of trains, but are also mechanically attached to each other by hook-like cell body structures [30]. In [40] we examined the hydrodynamics of sperm attachment and detachment from epithelia by allowing for forces due to dynamic elastic linkages between the actuated elastic flagellum and a planar wall. We will use the 3D extension described here, along with these dynamic linkages, to capture the complex binding interactions observed in some species [18, 24, 30, 31].

Our current model is a simplistic model chosen to compare with previous planar model results. For instance, the effect of a sperm head has not been included in our model, which would introduce drag at the base of the flagellum and could affect motility patterns particularly near surfaces (see the recent work of Ishimoto and Gaffney [23]). Moreover, some species have 
been shown experimentally to have three-dimensional sperm configurations that cannot be represented by simple planar forces described in our model. In fact, there is evidence that sperm flagella exhibit helical or non-planar waveforms in some settings [3, 36, 37, 51]. In Woolley and Osborn [50], the three-dimensional nature of hamster spermatozoa was determined to give rise to primarily clockwise torsional component, indicating that this came not from randomly applied (or external) forces but some structural feature of the flagellum itself. Additionally, the sea urchin behavior observed in some studies $[10,51]$ is thought to demonstrate a natural three-dimensional beat pattern which only reduces to a near-planar motion near surfaces. For these intrinsic three-dimensional waveforms, the method of regularized Stokeslets coupled to a Kirchhoff rod model [33] should be explored.

\section{Acknowledgements}

The work of JS, RC and LF was supported, in part, by the National Science Foundation grant DMS-104626.

\section{Conflict of interest statement}

The authors have no conflicts of interest to disclose.

[1] Arnold, V. I., 1989. Mathematical Methods of Classical Mechanics. Vol. 60 of Graduate Texts in Mathematics. Springer.

[2] Berg, H. C., 1993. Random walks in biology. Princeton University Press.

[3] Bishop, D. W., 1962. Sperm motility. Physiol. Rev 42 (1), 21. 
[4] Brokaw, C., 1965. Non-sinusoidal bending waves of sperm flagella. Journal of Experimental Biology 43 (1), 155-169.

[5] Brumley, D., Wan, K., Polin, M., Goldstein, R., 2014. Flagellar synchronization through direct hydrodynamic interactions. eLife 3, e02750.

[6] Chrispell, J., Fauci, L., Shelley, M., 2013. An actuated elastic sheet interacting with passive and active structures in a viscoelastic fluid. Phys Fluids 25, 013103-1-16.

[7] Corkidi, G., Taboada, B., Wood, C., Guerrero, A., Darszon, A., 2008. Tracking sperm in three-dimensions. Biochemical and Biophysical Research Communications 373 (1), 125-129.

[8] Cortez, R., 2001. The method of regularized stokeslets. SIAM Journal on Scientific Computing 23 (4), 1204-1225.

[9] Cortez, R., Fauci, L., Medovikov, A., 2005. The method of regularized stokeslets in three dimensions: analysis, validation, and application to helical swimming. Physics of Fluids (1994-present) 17 (3), 031504.

[10] Cosson, J., Huitorel, P., Gagnon, C., 2003. How spermatozoa come to be confined to surfaces. Cell Motility and the Cytoskeleton 54 (1), 56-63.

[11] Cummins, J., Woodall, P., 1985. On mammalian sperm dimensions. Journal of Reproduction and Fertility 75 (1), 153-175.

[12] David Eberly, Geometric Tools, L., 2014. Least squares fitting of data. http://www .geometrictools.com/Documentation/LeastSquaresFitting.pdf, accessed: 2014-01-30. 
[13] Elfring, G., Lauga, E., 2009. Hydrodynamic phase locking of swimming microorganisms. Phys Rev Lett 103, 088101.

[14] Fauci, L., 1990. Interaction of oscillating filaments: A computational study. J Comp Phys 86, 294-313.

[15] Fauci, L., Dillon, R., 2006. Biofluidmechanics of reproduction. Annu Rev Fluid Mech 38, 371-394.

[16] Fauci, L. J., McDonald, A., 1995. Sperm motility in the presence of boundaries. Bulletin of Mathematical Biology 57 (5), 679-699.

[17] Fauci, L. J., Peskin, C. S., 1988. A computational model of aquatic animal locomotion. Journal of Computational Physics 77 (1), 85-108.

[18] Fisher, H. S., Hoekstra, H. E., 2010. Competition drives cooperation among closely related sperm of deer mice. Nature 463 (7282), 801-803.

[19] Gibbons, B. H., Baccetti, B., Gibbons, I., 1985. Live and reactivated motility in the $9+0$ flagellum of anguilla sperm. Cell Motility 5 (4), 333-350.

[20] Gray, J., Hancock, G., 1955. The propulsion of sea-urchin spermatozoa. J Exp Biol 32, 802-814.

[21] Guerrero, A., Carneiro, J., Pimentel, A., Wood, C. D., Corkidi, G., Darszon, A., 2011. Strategies for locating the female gamete: the importance of measuring sperm trajectories in three spatial dimensions. Molecular Human Reproduction 17 (8), 511-523. 
[22] Ishijima, S., Sekiguchi, K., Hiramoto, Y., 1988. Comparative study of the beat patterns of american and asian horseshoe crab sperm: evidence for a role of the central pair complex in forming planar waveforms in flagella. Cell Motility and the Cytoskeleton 9 (3), 264-270.

[23] Ishimoto, K., Gaffney, E. A., 2014. A study of spermatozoan swimming stability near a surface. Journal of Theoretical Biology 360, 187-199.

[24] Johnston, S. D., Smith, B., Pyne, M., Stenzel, D., Holt, W. V., 2007. One-sided ejaculation of echidna sperm bundles. The American Naturalist 170 (6), E162-E164.

[25] Keppler, E., 1999. Aggregation of human sperm at higher temperature is due to hyperactivation. Systems Biology in Reproductive Medicine 42 (1), 35-39.

[26] Lin, J., Heuser, T., Song, K., Fu, X., Nicastro, D., 2012. One of the nine doublet microtubules of eukaryotic flagella exhibits unique and partially conserved structures. PloS One 7 (10), e46494.

[27] Lindemann, C. B., Orlando, A., Kanous, K. S., 1992. The flagellar beat of rat sperm is organized by the interaction of two functionally distinct populations of dynein bridges with a stable central axonemal partition. Journal of Cell Science 102 (2), 249-260.

[28] Llopis, I., Pagonabarraga, I., Lagomarsino, M. C., Lowe, C., 2013. Cooperative motion of intrinsic and actuated semiflexible swimmers. Physical Review E 87 (3), 032720. 
[29] Mettot, C., Lauga, E., 2011. Energetics of synchronized states in threedimensional beating flagella. Physical Review E 84 (6), 061905.

[30] Moore, H., Dvorakova, K., Jenkins, N., Breed, W., 2002. Exceptional sperm cooperation in the wood mouse. Nature 418 (6894), 174-177.

[31] Moore, H., Taggart, D., 1995. Sperm pairing in the opossum increases the efficiency of sperm movement in a viscous environment. Biology of Reproduction 52 (4), 947-953.

[32] Ohmuro, J., Ishijima, S., 2006. Hyperactivation is the mode conversion from constant-curvature beating to constant-frequency beating under a constant rate of microtubule sliding. Molecular Reproduction and Development 73 (11), 1412-1421.

[33] Olson, S., Lim, S., Cortez, R., 2013. Modeling the dynamics of an elastic rod with intrinsic curvature and twist using a regularized Stokes formulation. J. Comp. Phys. 238, 169-187.

[34] Olson, S. D., Suarez, S. S., Fauci, L. J., 2011. Coupling biochemistry and hydrodynamics captures hyperactivated sperm motility in a simple flagellar model. Journal of Theoretical Biology 283 (1), 203-216.

[35] Omoto, C. K., Gibbons, I. R., Kamiya, R., Shingyoji, C., Takahashi, K., Witman, G. B., 1999. Rotation of the central pair microtubules in eukaryotic flagella. Molecular Biology of the Cell 10 (1), 1-4.

[36] Phillips, D. M., 1972. Comparative analysis of mammalian sperm motility. The Journal of Cell Biology 53 (2), 561-573. 
[37] Rikmenspoel, R., 1965. The tail movement of bull spermatozoa: Observations and model calculations. Biophysical Journal 5 (4), 365-392.

[38] Serres, C., Escalier, D., David, G., 1984. Ultrastructural morphometry of the human sperm flagellum with a stereological analysis of the lengths of the dense fibres. Biology of the Cell 49 (2), 153-161.

[39] Shingyoji, C., Katada, J., Takahashi, K., Gibbons, I., 1991. Rotating the plane of imposed vibration can rotate the plane of flagellar beating in sea-urchin sperm without twisting the axoneme. Journal of Cell Science 98 (2), 175-181.

[40] Simons, J., Olson, S., Cortez, R., Fauci, L., 2014. The dynamics of sperm detachment from epithelium in a coupled fluid-biochemical model of hyperactivated motility. Journal of Theoretical Biology 354, 81-94.

[41] Smith, D., Gaffney, E., Gadêlha, H., Kapur, N., Kirkman-Brown, J., 2009. Bend propagation in the flagella of migrating human sperm, and its modulation by viscosity. Cell Motility and the Cytoskeleton 66 (4), $220-236$.

[42] Su, T.-W., Choi, I., Feng, J., Huang, K., McLeod, E., Ozcan, A., 2013. Sperm trajectories form chiral ribbons. Scientific Reports 3.

[43] Su, T.-W., Xue, L., Ozcan, A., 2012. High-throughput lensfree 3D tracking of human sperms reveals rare statistics of helical trajectories. Proceedings of the National Academy of Sciences 109 (40), 16018-16022.

[44] Suarez, S., Katz, D., Owen, D., Andrew, J., Powell, R., 1991. Evidence 
for the function of hyperactivated motility in sperm. Biology of Reproduction 44 (2), 375-381.

[45] Suarez, S. S., 2008. Control of hyperactivation in sperm. Human Reproduction Update 14 (6), 647-657.

[46] Taylor, G., 1951. Analysis of the swimming of microscopic organisms. Proc Roy Soc Lond Ser A 209, 447-461.

[47] Teran, J., Fauci, L., Shelley, M., 2010. Viscoelastic fluid response can increase the speed of a free swimmer. Phys Rev Lett 104, 038101-4.

[48] Vernon, G., Woolley, D., 2004. Basal sliding and the mechanics of oscillation in a mammalian sperm flagellum. Biophys. J. 85 (6), 3934-3944.

[49] Woolley, D., Crockett, R., Groom, W., Revell, S., 2009. A study of synchronisation between the flagella of bull spermatozoa, with related observations. J Exp Biol 212, 2215-2223.

[50] Woolley, D., Osborn, I., 1984. Three-dimensional geometry of motile hamster spermatozoa. Journal of Cell Science 67 (1), 159-170.

[51] Woolley, D., Vernon, G., 2001. A study of helical and planar waves on sea urchin sperm flagella, with a theory of how they are generated. Journal of Experimental Biology 204 (7), 1333-1345.

[52] Yanagimachi, R., 1970. The movement of golden hamster spermatozoa before and after capacitation. Journal of Reproduction and Fertility 23 (1), 193-196. 
${ }_{546}$ [53] Yang, Y., Elgeti, J., Gompper, G., 2008. Cooperation of sperm in two dimensions: synchronization, attraction, and aggregation through hy-

548 drodynamic interactions. Phys Rev E 78, 061903-1-9. 


\section{7. Table}

\begin{tabular}{llll}
\hline Parameter & Description & Value & Remark/Reference(s) \\
\hline$\epsilon$ & Regularization parameter & $1.3 \mu \mathrm{m}$ & See [8] \\
& Diameter of sperm flagellum & $0.5 \mu \mathrm{m}$ & {$[38]$} \\
$\mu$ & Viscosity (water) & $10^{-3} \mathrm{~kg} \mathrm{~m}^{-1} \mathrm{~s}^{-1}$ & \\
$\Delta s$ & Spatial (arc length) discretization & $1 \mu \mathrm{m}$ & \\
$L$ & Flagellum length & $100 \mu \mathrm{m}$ & {$[11]$} \\
$b$ & Amplitude & $10-25 \mu \mathrm{m}$ & {$[32,41]$} \\
$\kappa$ & Wavenumber & $2 \pi / L$ & \\
$\omega$ & Frequency & $20 \pi(10 \mathrm{~Hz})$ & {$[32,41]$} \\
$S_{t}$ & Tensile stiffness & $2 \mathrm{pN} \mu \mathrm{m}^{-3}$ & {$[40]$} \\
$S_{p}$ & Planar restriction stiffness & $0.001-1 \mathrm{pN}^{-3} \mathrm{~m}^{-3}$ & This work \\
$S_{b}$ & Planar bending stiffness & $10 \mathrm{pN} \mu \mathrm{m}^{-3}$ & {$[34]$} \\
$S_{r}$ & Repulsion stiffness & $5 \mathrm{aN} \mu \mathrm{m}^{-3}$ & This work \\
$d$ & Repulsion length & $3 \mu \mathrm{m}$ & Set to be $>2 \delta$ \\
\hline
\end{tabular}

Table 1: Parameter values used in models. Sperm parameters were set to that of a typical mammalian sperm (see [11]). The regularized delta function for the method of regularized Stokeslets is the same as in [34]. Note that in this framework, we are using force and energy densities, so stiffness constants include a factor of $\mu \mathrm{m}^{-3}$. 


\section{Figures}

Figure 1: Schematic of the physical domain of the discretized preferred configuration of the flagellum, with a sinusoidal waveform of amplitude $b$ and an arc length along the flagellum ranging from $s=0$ to $s=L$. Each point $\mathbf{X}_{k}$ experiences tensile forces from interactions with neighboring points, and bending forces that actuate the motion of the flagellum. The points have a preferred spacing of $\Delta s$.

Figure 2: Example of a least squares fit plane to a flagellum given by data $\mathbf{X}_{k}$ : (a) shows the original data, (b) shows the centered data with the least squares fit plane, and (c) shows the data in the flagellum frame of reference with a least squares fit plane $\hat{z}=0$. Dots represent the head of the sperm body, with darker shading showing the parts of the flagellum that are underneath the flagellar plane.

Figure 3: Initial configurations of sperm flagella. In simulations with a single sperm, the black curve in (a)-(c) is used, and has a fully threedimensional configuration. For two sperm coplanar simulations, we consider the flagella in panels (a)-(c), and project the black curve in (a)-(c) onto the plane $z=0$ so both sperm are in the plane $z=0$. For two sperm simulations with a non-planar perturbation, we use the red and black curves as shown in panels (a)-(c). For two sperm with initial parallel flagellar planes, we use the configurations in panels (d)-(f). All units are in $\mu \mathrm{m}$. These small amplitude initial configurations are chosen for simplicity, and the sperm shape at later times is a sinusoidal form with an amplitude near $10 \mu \mathrm{m}$, coming from the 
energies defined in Section 2.1.

Figure 4: Comparison of maximal deviations from the flagellar plane over time for various choices of planar restriction stiffness parameter $S_{p}$. These deviations were calculated for a single sperm with the initial threedimensional configuration given by the black curve in Figure 3, using the symmetric preferred curvature motility behavior.

Figure 5: Trajectories of the head points of two symmetrically beating sperm with different planar initial conditions. Triangles represent final positions at time $t=25 \mathrm{~s}$, and the starting position for the sperm heads is at $x=0$. Units are $\mu \mathrm{m}$. Panel (a) initializes both flagella as coplanar in the plane $z=0$ with initial conditions corresponding to the projections of the configurations in Figures 3a-3c onto $z=0$. Panel (b) initializes both flagella in parallel planes in $y$ with initial conditions shown in Figures 3d3f. Note that coplanar flagella attract, whereas parallel planar flagella repel. The Movies M2 and M3 show the swimming behavior of the trajectories in (a) and (b), respectively, over time.

Figure 6: Average path velocities and minimum distance (magenta curves) between flagella over time for symmetric swimmers with planar restriction stiffness $S_{p}=1$. Panel (a) shows velocities from the initial parallel configurations in Figure 3d-3f, corresponding to the trajectories in Figure 5b. Panel (b) shows velocities from the same initial parallel configurations, except the parallel planes are 10 times closer ( $5 \mu \mathrm{m}$ apart). To demonstrate at- 
traction versus repelling swimming behavior, both parallel plane simulations were run without any repulsion forces $\mathbf{g}_{k}^{j}$ that might interfere with trajectories. Panel (c) shows velocities from the coplanar initial configurations given by the projections of the configurations in Figure 3 onto the plane $z=0$, corresponding to the trajectories in Figure 5a. Panel (d) shows velocities from the non-planar initial configurations shown in Figure 3, corresponding to the trajectories in Figure 8a. Both panels (c) and (d) show simulations run with repulsion forces $\mathbf{g}_{k}^{j}$ present to prevent flagella from overlapping unrealistically.

Figure 7: Example of the positions of two symmetrically beating sperm at $t=0.1,5.1$, and 10.1 seconds with planar restriction stiffness $S_{p}=1$. These are snapshots of sperm locations from the trajectories shown in Figure 8a. Units are $\mu \mathrm{m}$. Initial configurations are shown in Figure 3. The Movie M4 shows the swimming behavior of this simulation over time.

Figure 8: Example of trajectories of the head points of two symmetrically beating sperm with different planar restriction stiffnesses $S_{p}$, over 25 seconds. Triangles represent final positions, starting position for sperm heads is at $x=0$. Units are $\mu \mathrm{m}$. Initial configurations are shown in Figure 3. The Movies M4 and M5 show the swimming behavior of the trajectories in (a) and (b), respectively, over time.

Figure 9: Example of trajectories of the head points of weakly asymmetrically beating sperm using a planar restriction stiffness of $S_{p}=1$, traced out over the times indicated with the initial configurations shown in Figures 
625 3a-3c. "Zigzag" in the trajectories demonstrates the lateral displacement of 626 the head. Triangles (partially occluded) are placed at the end of trajectories, ${ }_{627}$ starting position for sperm heads is at $x=0$. Units are in $\mu \mathrm{m}$. The Movies ${ }_{628}$ M1 and M6 show the swimming behaviors of (a) and (b), respectively, over 629 time. 


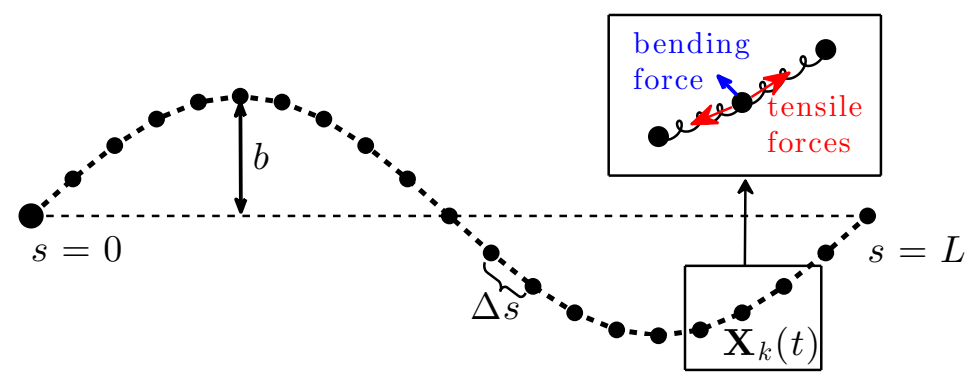

Figure 1: Schematic of the physical domain of the discretized preferred configuration of the flagellum, with a sinusoidal waveform of amplitude $b$ and an arc length along the flagellum ranging from $s=0$ to $s=L$. Each point $\mathbf{X}_{k}$ experiences tensile forces from interactions with neighboring points, and bending forces that actuate the motion of the flagellum. The points have a preferred spacing of $\Delta s$. 


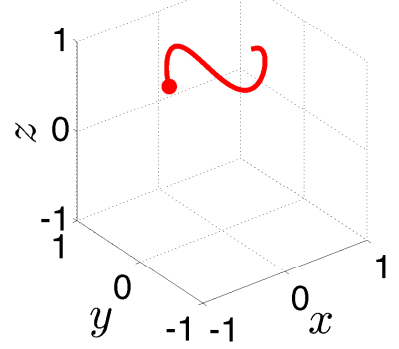

(a) $\mathbf{X}_{k}$

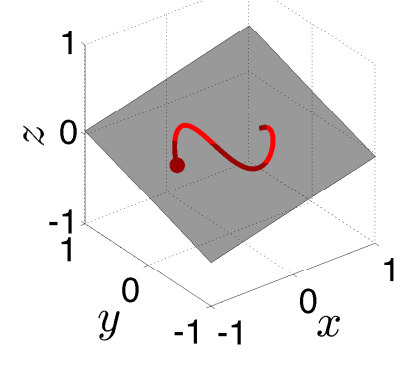

(b) $\mathbf{X}_{k}-\overline{\mathbf{X}}$.

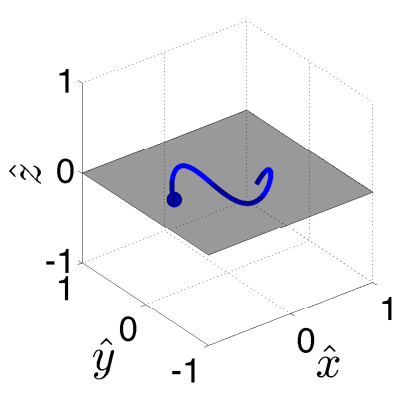

(c) $\hat{\mathbf{X}}_{k}$.

Figure 2: Example of a least squares fit plane to a flagellum given by data $\mathbf{X}_{k}$ : (a) shows the original data, (b) shows the centered data with the least squares fit plane, and (c) shows the data in the flagellum frame of reference with a least squares fit plane $\hat{z}=0$. Dots represent the head of the sperm body, with darker shading showing the parts of the flagellum that are underneath the flagellar plane. 


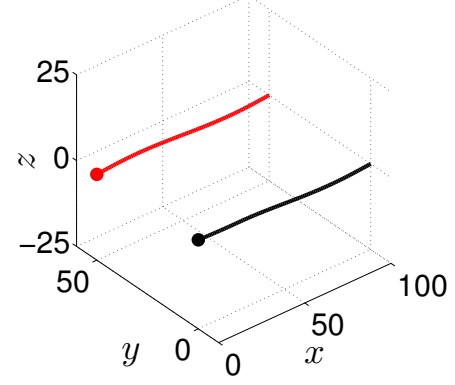

(a) $3 \mathrm{D}$ view.

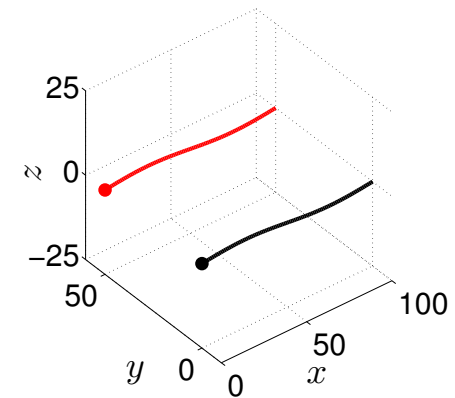

(d) $3 \mathrm{D}$ view.

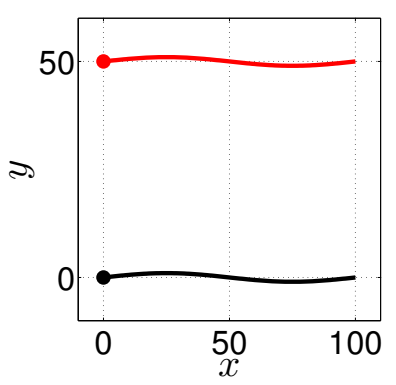

(b) Projection onto $z=0$.
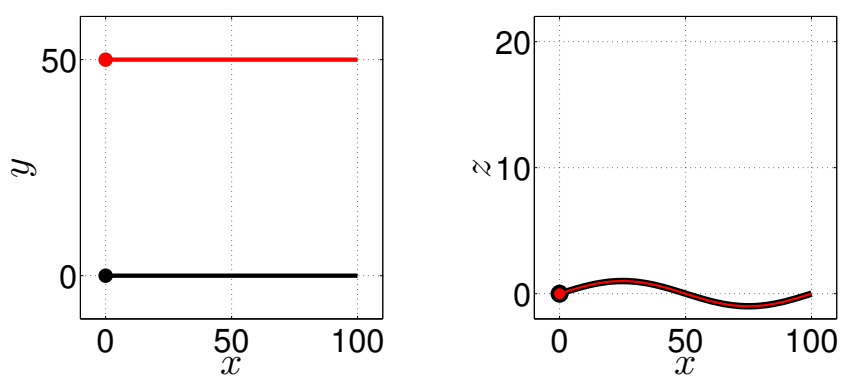

(e) Projection onto $z=0$. (f) Projection onto $y=0$.

Figure 3: Initial configurations of sperm flagella. In simulations with a single sperm, the black curve in (a)-(c) is used, and has a fully three-dimensional configuration. For two sperm coplanar simulations, we consider the flagella in panels (a)-(c), and project the black curve in (a)-(c) onto the plane $z=0$ so both sperm are in the plane $z=0$. For two sperm simulations with a non-planar perturbation, we use the red and black curves as shown in panels (a)-(c). For two sperm with initial parallel flagellar planes, we use the configurations in panels (d)-(f). All units are in $\mu \mathrm{m}$. These small amplitude initial configurations are chosen for simplicity, and the sperm shape at later times is a sinusoidal form with an amplitude near $10 \mu \mathrm{m}$, coming from the energies defined in Section 2.1. 


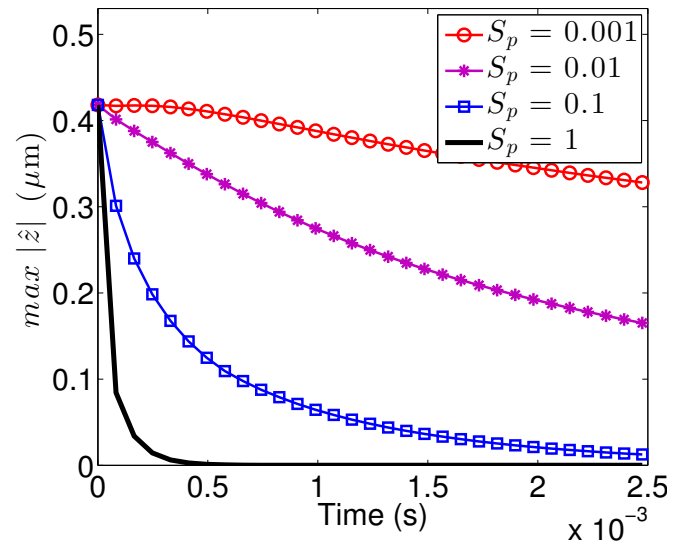

Figure 4: Comparison of maximal deviations from the flagellar plane over time for various choices of planar restriction stiffness parameter $S_{p}$. These deviations were calculated for a single sperm with the initial three-dimensional configuration given by the black curve in Figure 3, using the symmetric preferred curvature motility behavior.

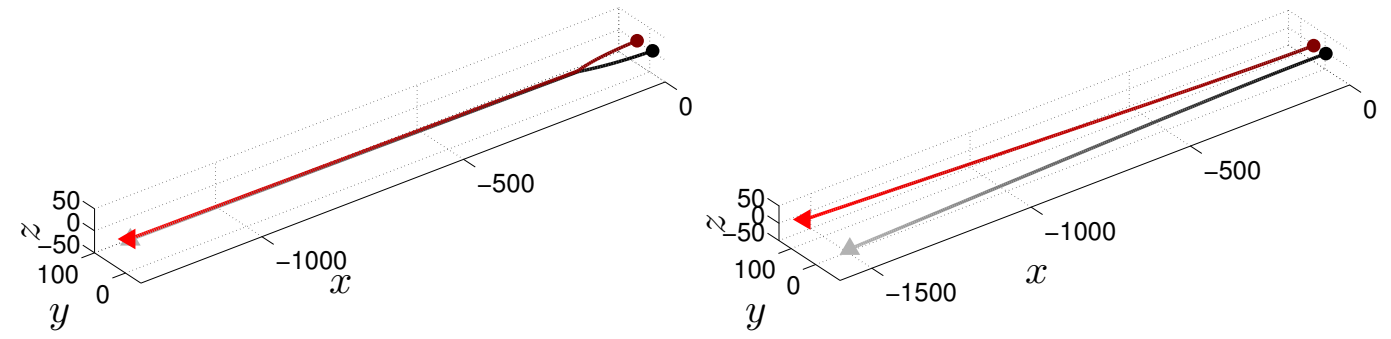

(a) Coplanar.

(b) Parallel initial flagellar planes.

Figure 5: Trajectories of the head points of two symmetrically beating sperm with different planar initial conditions. Triangles represent final positions at time $t=25 \mathrm{~s}$, and the starting position for the sperm heads is at $x=0$. Units are $\mu \mathrm{m}$. Panel (a) initializes both flagella as coplanar in the plane $z=0$ with initial conditions corresponding to the projections of the configurations in Figures 3a-3c onto $z=0$. Panel (b) initializes both flagella in parallel planes in $y$ with initial conditions shown in Figures $3 \mathrm{~d}-3 \mathrm{f}$. Note that coplanar flagella attract, whereas parallel planar flagella repel. The Movies M2 and M3 show the swimming behavior of the trajectories in (a) and (b), respectively, over time. 


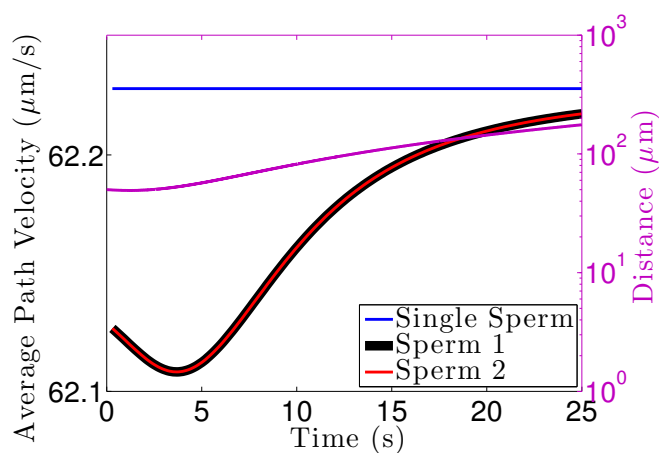

(a) Parallel, $50 \mu \mathrm{m}$ apart.

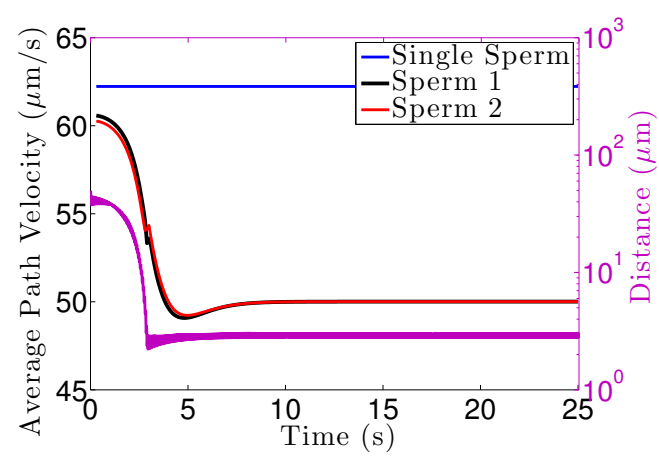

(c) Coplanar.

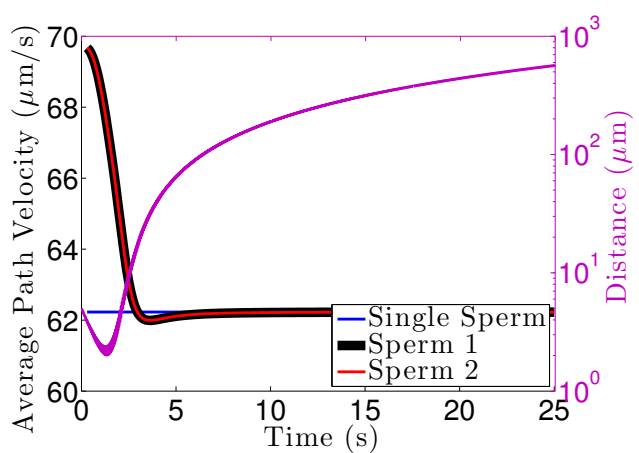

(b) Parallel, $5 \mu \mathrm{m}$ apart.

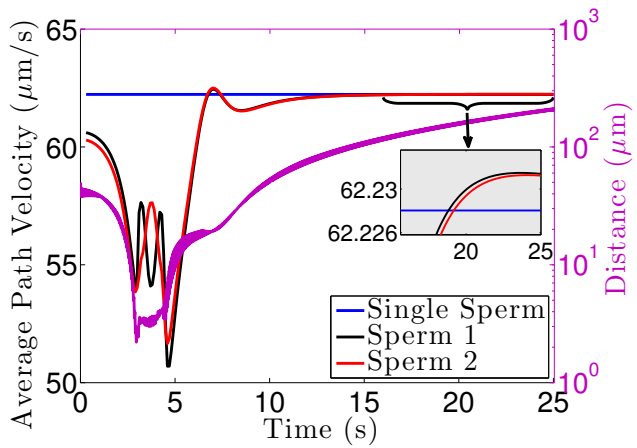

(d) Non-planar.

Figure 6: Average path velocities and minimum distance (magenta curves) between flagella over time for symmetric swimmers with planar restriction stiffness $S_{p}=1$. Panel (a) shows velocities from the initial parallel configurations in Figure 3d-3f, corresponding to the trajectories in Figure 5b. Panel (b) shows velocities from the same initial parallel configurations, except the parallel planes are 10 times closer ( $5 \mu \mathrm{m}$ apart). To demonstrate attraction versus repelling swimming behavior, both parallel plane simulations were run without any repulsion forces $\mathbf{g}_{k}^{j}$ that might interfere with trajectories. Panel (c) shows velocities from the coplanar initial configurations given by the projections of the configurations in Figure 3 onto the plane $z=0$, corresponding to the trajectories in Figure 5a. Panel (d) shows velocities from the non-planar initial configurations shown in Figure 3, corresponding to the trajectories in Figure 8a. Both panels (c) and (d) show simulations run with repulsion forces $\mathbf{g}_{k}^{j}$ present to prevent flagella from overlapping unrealistically. 


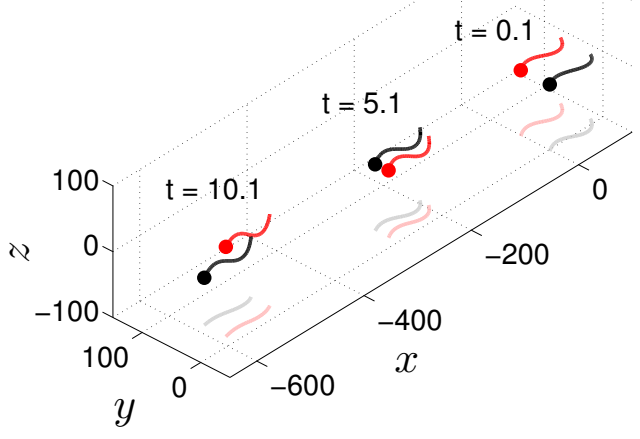

(a) $3 \mathrm{D}$ view.

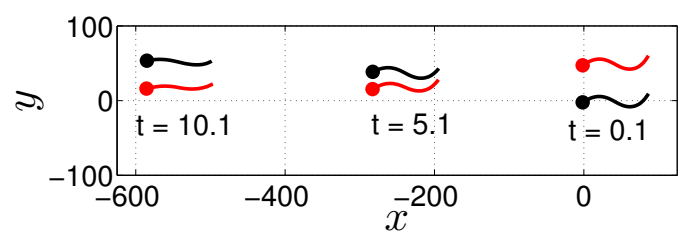

(c) $x-y$ view.

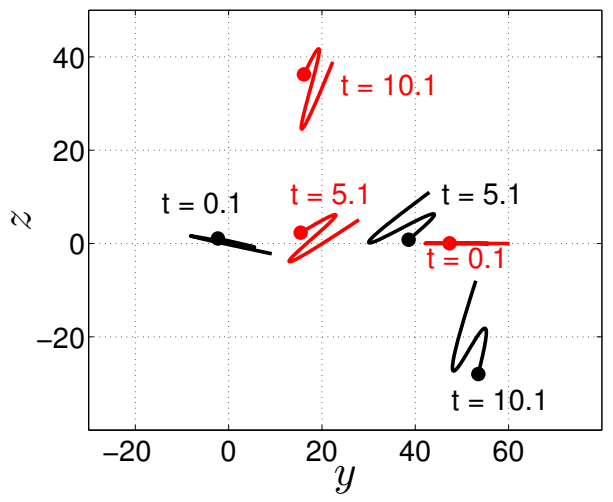

(b) $y-z$ view.

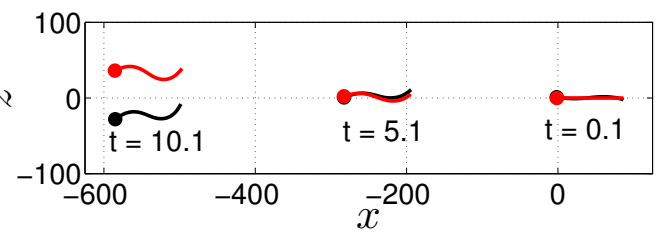

(d) $x-z$ view.

Figure 7: Example of the positions of two symmetrically beating sperm at $t=0.1,5.1$, and 10.1 seconds with planar restriction stiffness $S_{p}=1$. These are snapshots of sperm locations from the trajectories shown in Figure 8a. Units are $\mu \mathrm{m}$. Initial configurations are shown in Figure 3. The Movie M4 shows the swimming behavior of this simulation over time. 


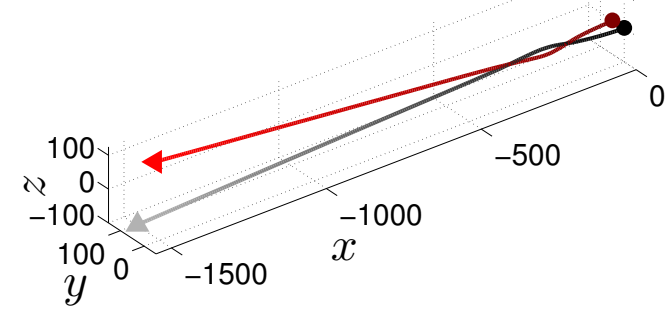

(a) $S_{p}=1$.

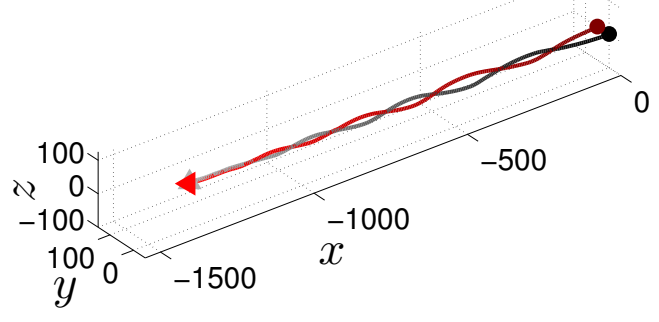

(b) $S_{p}=0.01$.

Figure 8: Example of trajectories of the head points of two symmetrically beating sperm with different planar restriction stiffnesses $S_{p}$, over 25 seconds. Triangles represent final positions, starting position for sperm heads is at $x=0$. Units are $\mu \mathrm{m}$. Initial configurations are shown in Figure 3. The Movies M4 and M5 show the swimming behavior of the trajectories in (a) and (b), respectively, over time. 


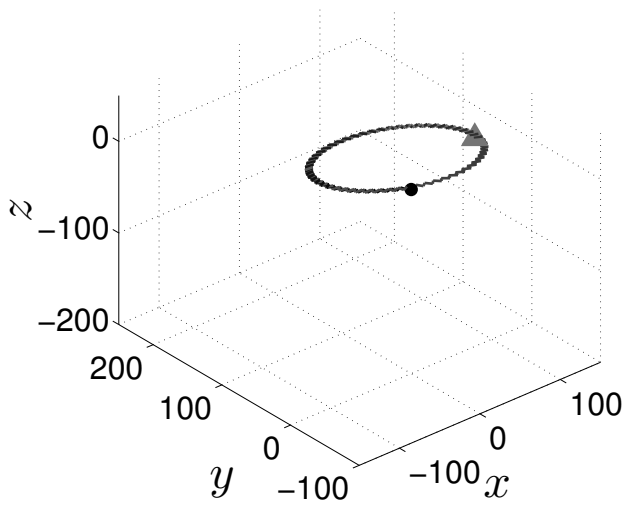

(a) Single sperm, $t=0$ to $10 \mathrm{~s}$.

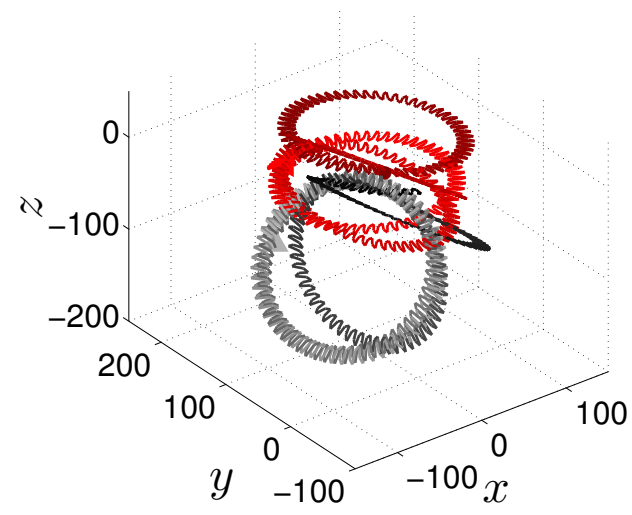

(b) Two sperm, $t=0$ to $25 \mathrm{~s}$.

Figure 9: Example of trajectories of the head points of weakly asymmetrically beating sperm using a planar restriction stiffness of $S_{p}=1$, traced out over the times indicated with the initial configurations shown in Figures 3a-3c. "Zigzag" in the trajectories demonstrates the lateral displacement of the head. Triangles (partially occluded) are placed at the end of trajectories, starting position for sperm heads is at $x=0$. Units are in $\mu \mathrm{m}$. The Movies M1 and M6 show the swimming behaviors of (a) and (b), respectively, over time. 


\section{Movie Legend}

Movie M1: A weakly asymmetric swimmer starting with a perturbed planar initial configuration (see black curve in Figures 3a-3c), with planar stiffness constant $S_{p}=1$. The sperm travels in a circular path, recovering from its non-planar configuration quickly. Gray curve shows the projection ("shadow") of the flagellum on the plane $z=-100$. Units are in microns. Length of simulation is 25 seconds.

Movie M2: Two symmetric swimmers with a coplanar initial configuration (see Figures $3 \mathrm{a}-3 \mathrm{c}$ ), with planar stiffness constant $S_{p}=1$. The two swimmers attract over time. Light shaded curves show the projections ("shadows") of the flagella on the plane $z=-100$. Units are in microns. Length of simulation is 25 seconds.

Movie M3: Two symmetric swimmers with a parallel planar initial configuration (see Figures 3d-3f), with planar stiffness constant $S_{p}=1$. The two swimmers repel over time. Light shaded curves show the projections ("shadows") of the flagella on the plane $z=-100$. Units are in microns. Length of simulation is 25 seconds.

Movie M4: Two symmetric swimmers with a perturbed planar initial configuration (see Figures 3a-3c), with planar stiffness constant $S_{p}=1$. The two swimmers "weave" near each other before swimming apart. Light shaded curves show the projections ("shadows") of the flagella on the plane $z=-150$. Units are in microns. Length of simulation is 25 seconds. 
656

Movie M5: Two symmetric swimmers with a perturbed planar initial configuration (see Figures 3a-3c), with planar stiffness constant $S_{p}=0.01$. The two swimmers "weave" near each other for a more prolonged period than in Movie M4 because of a weaker stiffness constant. Light shaded curves show the projections ("shadows") of the flagella on the plane $z=-100$. Units are in microns. Length of simulation is 25 seconds.

Movie M6: Two weakly asymmetric swimmers with a perturbed planar initial configuration (see Figures 3a-3c), with planar stiffness constant $S_{p}=1$. The two swimmers move in curved "slinky-like" trajectories over time. Light shaded curves show the projections ("shadows") of the flagella on the plane $z=-250$. Units are in microns. Length of simulation is 25 seconds.

Movie M7: Two strongly asymmetric swimmers with a perturbed planar initial configuration (see Figures 3a-3c), with planar stiffness constant $S_{p}=$ 1. The two swimmers move in curved paths over time with a smaller radius of curvature due to high asymmetry, resulting in "ball-like" trajectories. Light shaded curves show the projections ("shadows") of the flagella on the plane $z=-100$. Units are in microns. Length of simulation is 25 seconds. 


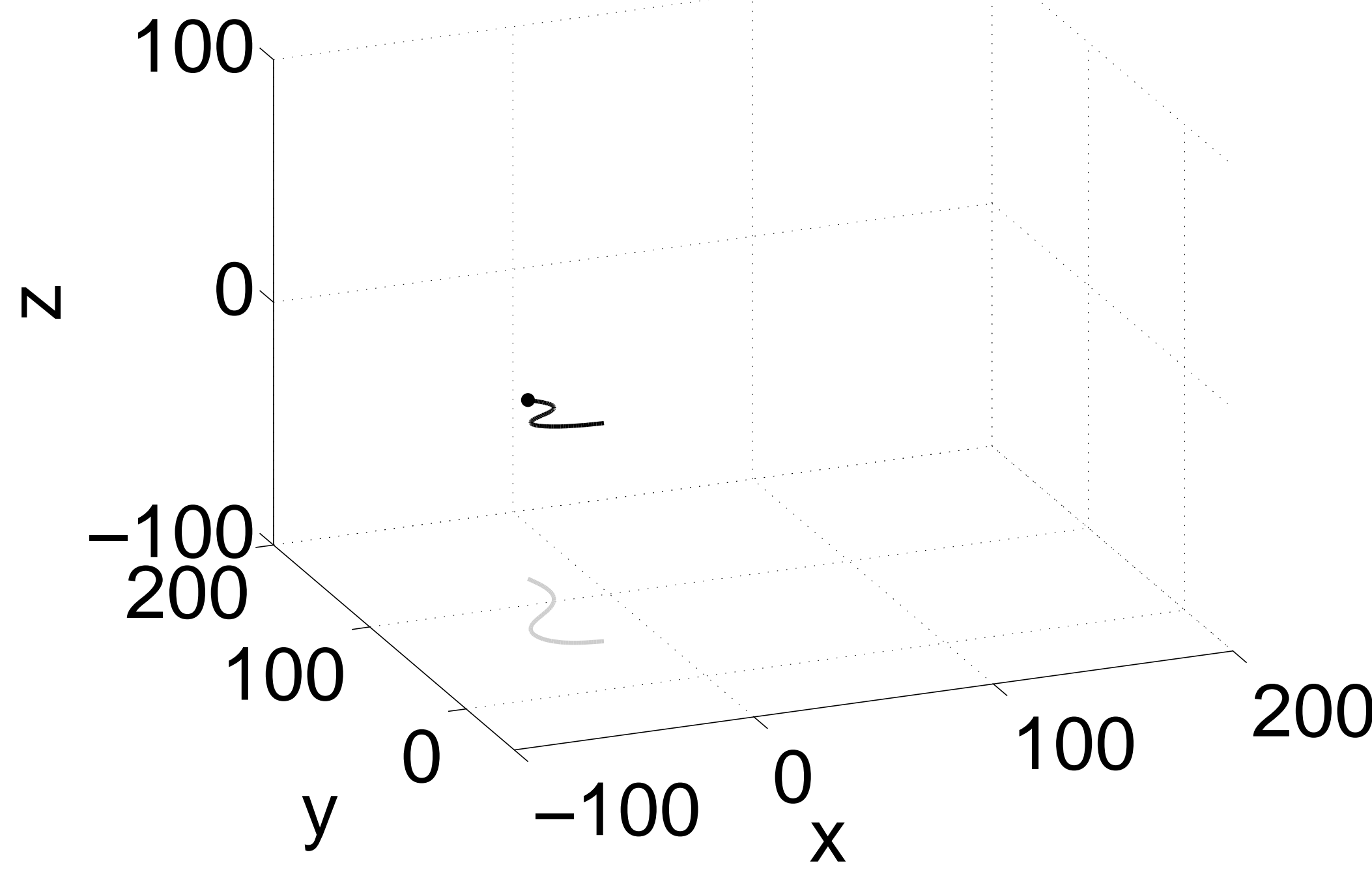




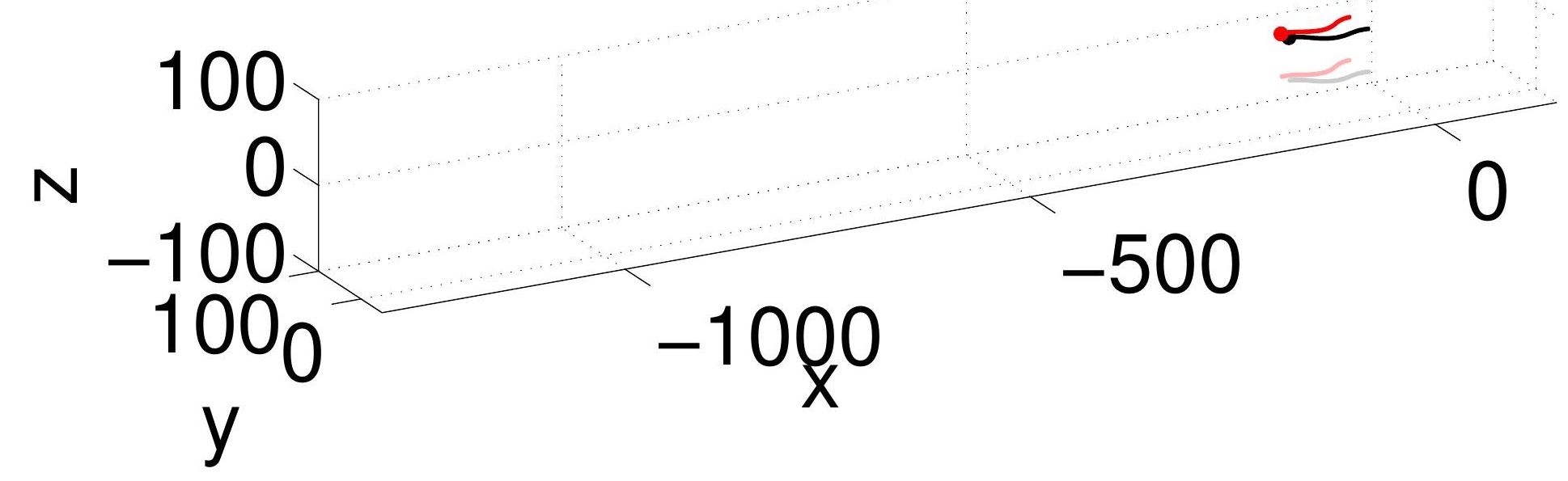


$\sigma$

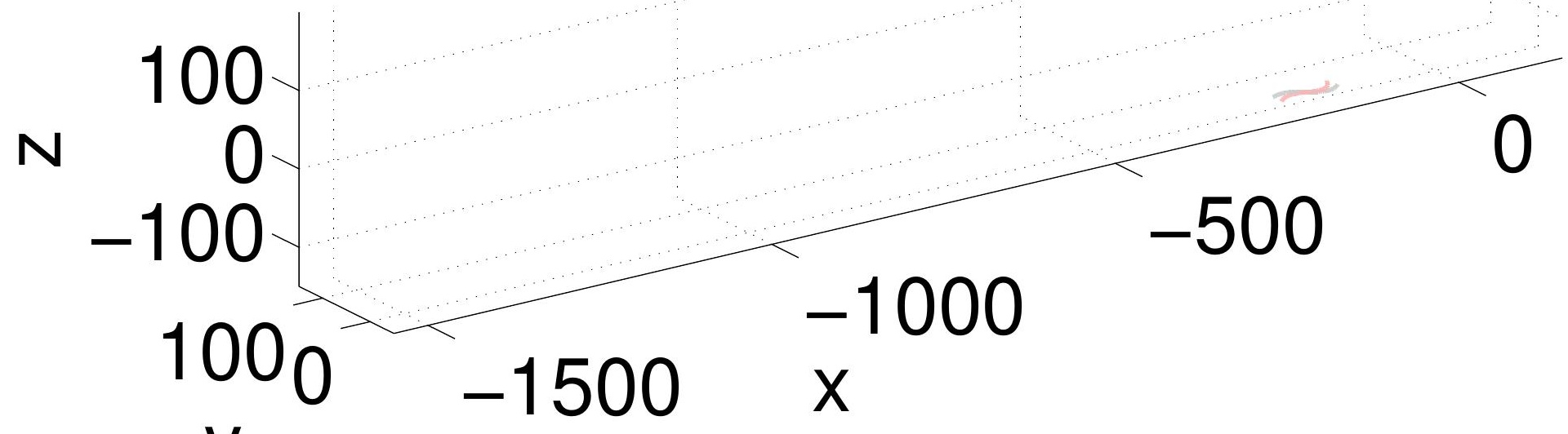




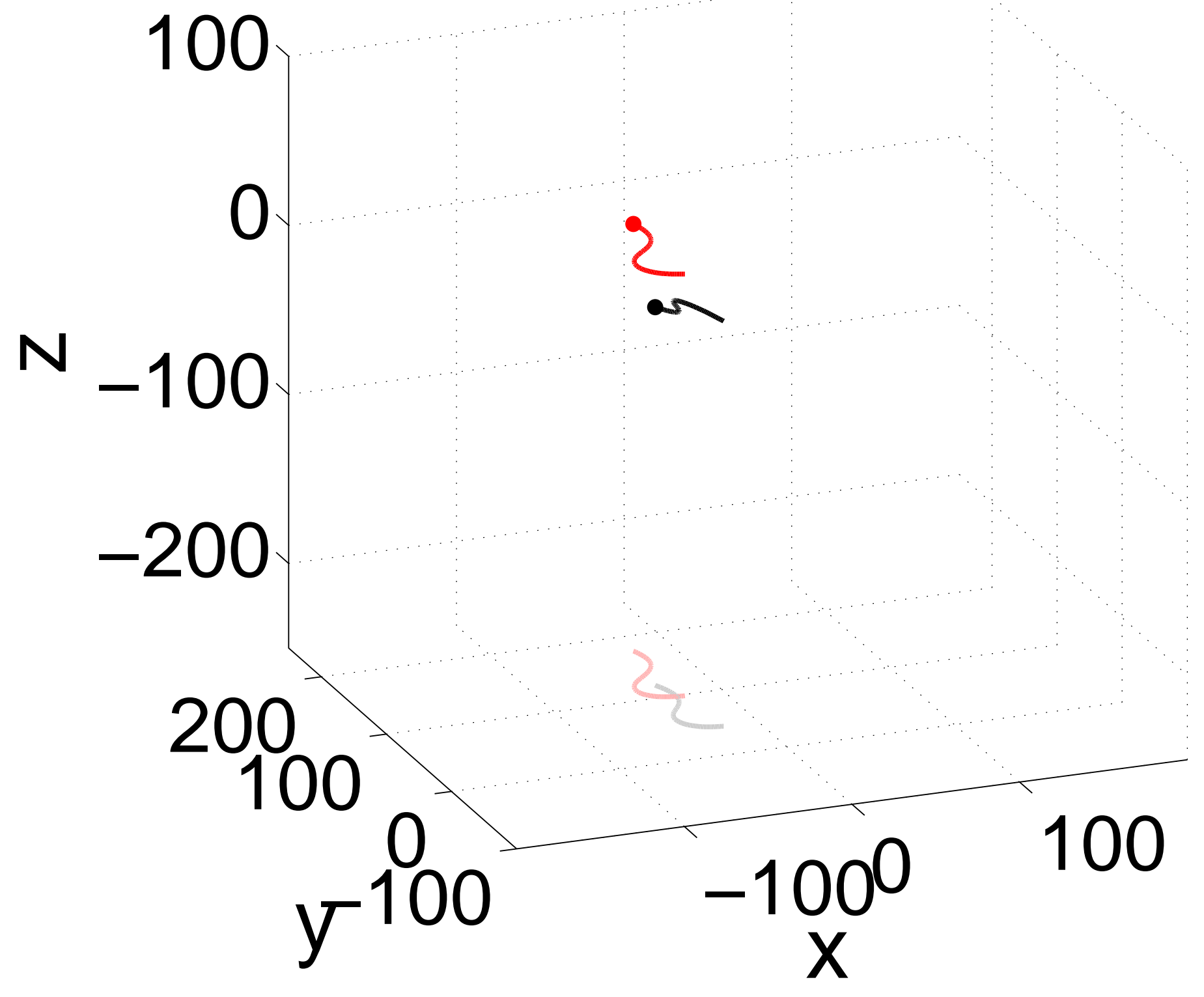




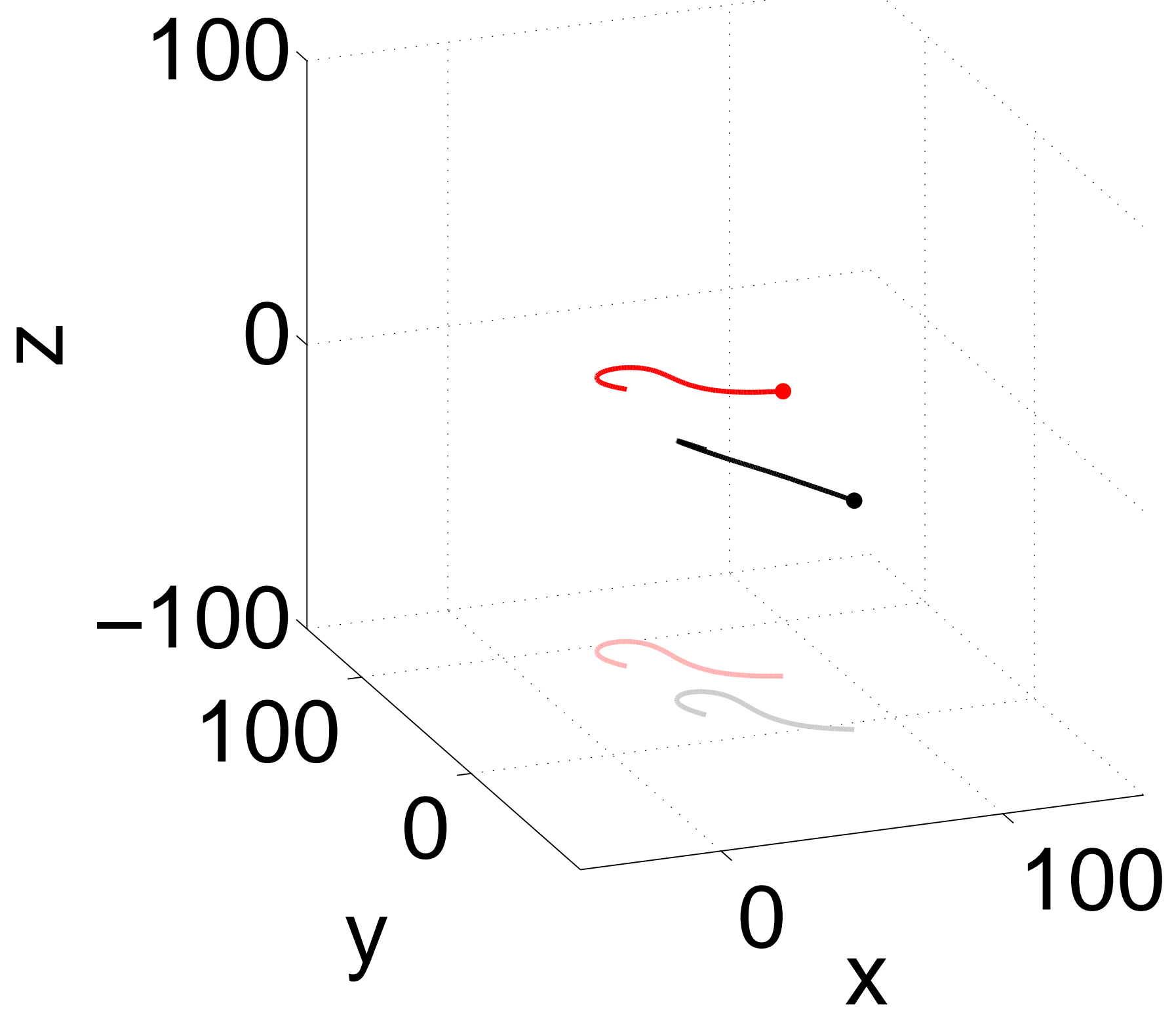

